\title{
A review of Diplommatina species in eastern Thailand with the descriptions of five new species
}

\author{
Pongrat Dumrongrojwattana ${ }^{\ddagger}$, Chanakarn Kamtuptim ${ }^{\ddagger}$, Koraon Wongkamhaeng $\S$ \\ ‡ Department of Biology, Faculty of Science, Burapha University, Chon Buri, Thailand \\ $\S$ Kasetsart University, Bangkok, Thailand
}

Corresponding author: Pongrat Dumrongrojwattana (pongrat@buu.ac.th)

Academic editor: Kenneth Hayes

Received: 17 Aug 2020 | Accepted: 21 Sep 2020 | Published: 06 Oct 2020

Citation: Dumrongrojwattana P, Kamtuptim C, Wongkamhaeng K (2020) A review of Diplommatina species in eastern Thailand with the descriptions of five new species. Biodiversity Data Journal 8: e57689.

https://doi.org/10.3897/BDJ.8.e57689

ZooBank: urn:Isid:zoobank.org:pub:C64E4072-1516-4214-9AA9-29407AFD28F8

\begin{abstract}
\section{Background}

Microsnails in the genus Diplommatina Benson, 1849 from eastern Thailand are revised, based on the collection of the Zoological Research Collection, Burapha University, Chonburi Province, Thailand and on recently-collected materials.
\end{abstract}

\section{New information}

Five new species, Diplommatina burapha sp. n., $D$. chadathongae sp. n., $D$. chantaburiensis sp. n., D. fusiformis sp. n. and $D$. khwantongae sp. n., are described as new to science. The geographic distribution of these eastern species is presented.

\section{Keywords}

Diplommatina, Diplommatinidae, taxonomy, Thailand 


\section{Introduction}

Terrestrial microsnails in the genus Diplommatina Benson, 1849 show some of the greatest diversity and are distributed in southern, south-eastern and eastern Asia. At present, a total of 18 Diplommatina species have been reported from Thailand. Of the 18 described species, ten $(55.55 \%)$ were recorded from the south and seven $(38.9 \%)$ from the north, whereas only one species, D. crispata khaochamaoensis Panha et al., 1998, was described from Khaochamao National Park, Chantaburi Province (Maassen 2001, Panha 1996, Panha and Burch 1996, Panha and Burch 2001, Panha and Burch 2005, Panha et al. 1998, Tongkerd et al. 2013). This study is focused on a survey of microsnail diversity recorded in eastern Thailand since 2005 and focused on five species of the genus Diplommatina ( $D$. burapha sp. n., $D$. chadathongae sp. n., $D$. fusiformis sp. n., $D$. khwantongae sp. $\mathrm{n}$. and $D$. khaowongensis) as new to science, based on the external shell characters. The geographic distribution of these eastern Thai species is also presented.

\section{Materials and methods}

The specimens from the Zoological Research Collection of Burapha University (ZRCBUU) which have been collected since 2005 and recently obtained shell specimens from the field survey were studied. Shells were collected by hand searching and leaf litter sieving in several areas in eastern Thailand (Fig. 1 and Table 1). Empty shells were cleaned with tap water and a fine hairbrush and then air-dried. Only live specimens of one species, Diplommatina khwantongae were collected. Digital photographs of shells were obtained using a Canon MP-E 65 Macro lens camera. Shell measurements (in $\mathrm{mm}$ ), including shell height $(\mathrm{SH})$, shell width $(\mathrm{SW})$, aperture height $(\mathrm{AH})$ and aperture width $(\mathrm{AW})$, were determined using ImageJ software. Taxonomic identification and shell terminology (e.g. whorl number and shell sculpture) of the specimens were recored according to Panha et al. (1998) and Tongkerd et al. (2013). Type specimens were deposited in the collection of the Zoological Research Collection of Burapha University (ZRCBUU), Chon Buri, Thailand.

Table 1.

List and coordination of collecting sites.

\begin{tabular}{|l|l|l|l|}
\hline Province & Code & Location & Coordination (Lat. Long) \\
\hline Chonburi & CHON 001 & Khaocha-ang Pluangtong & $13^{\circ} 11^{\prime} 56.4^{\prime \prime} \mathrm{N} 101^{\circ} 34^{\prime} 47.0^{\prime \prime} \mathrm{E}$ \\
\hline & CHON 002 & Khao Mhe Cave & $13^{\circ} 09^{\prime} 04.0^{\prime \prime} \mathrm{N} 101^{\circ} 35^{\prime} 35.0^{\prime \prime} \mathrm{E}$ \\
\hline & CHON 003 & Lublae Cave & $13^{\circ} 09^{\prime} 09.0^{\prime \prime} \mathrm{N} 101^{\circ} 35^{\prime} 52.1^{\prime \prime} \mathrm{E}$ \\
\hline Rayong & RA 001 & Khao Pratun & $13^{\circ} 07^{\prime} 36.9^{\prime \prime} \mathrm{N} 101^{\circ} 35^{\prime} 56.6^{\prime \prime} \mathrm{E}$ \\
\hline & RA 002 & Wat Tam Wattana Mongkol & $13^{\circ} 05^{\prime} 49.4^{\prime \prime} \mathrm{N} 101^{\circ} 36^{\prime} 25.1^{\prime \prime} \mathrm{E}$ \\
\hline & RA 003 & Pradoo Cave & $13^{\circ} 05^{\prime} 40.8^{\prime \prime} \mathrm{N} 101^{\circ} 36^{\prime} 25.1^{\prime \prime} \mathrm{E}$ \\
\hline & RA 004 & Khao Loi Cave & $13^{\circ} 03^{\prime} 26.8^{\prime \prime} \mathrm{N} 101^{\circ} 36^{\prime} 26.2^{\prime \prime} \mathrm{E}$ \\
\hline & RA 005 & Wat Tam Khaobote & $13^{\circ} 02^{\prime} 14.5^{\prime \prime} \mathrm{N} 101^{\circ} 38^{\prime} 04.4^{\prime \prime} \mathrm{E}$ \\
\hline
\end{tabular}




\begin{tabular}{|c|c|c|c|}
\hline Province & Code & Location & Coordination (Lat. Long) \\
\hline & RA 006 & Wat Tam Suwannaphupha & $12^{\circ} 59^{\prime} 20.2^{\prime \prime} \mathrm{N} 101^{\circ} 39^{\prime} 36.0^{\prime \prime} \mathrm{E}$ \\
\hline & RA 007 & Samnaksong Tam Naeramitr & $12^{\circ} 58^{\prime} 13.7^{\prime \prime} \mathrm{N} 101^{\circ} 39^{\prime} 45.7^{\prime \prime} \mathrm{E}$ \\
\hline & RA 008 & Wat Tam Rakangtong & $12^{\circ} 45^{\prime} 48.3^{\prime \prime} \mathrm{N} 101^{\circ} 47^{\prime} 47.2^{\prime \prime} \mathrm{E}$ \\
\hline & RA 009 & Wat Rattanatrikoon & $12^{\circ} 56^{\prime} 53.1^{\prime \prime} \mathrm{N} 101^{\circ} 46^{\prime} 37.1^{\prime \prime E}$ \\
\hline \multirow[t]{2}{*}{ Chantaburi } & СТВ 001 & Wat Tam Khaowong & $14^{\circ} 35^{\prime} 15.5^{\prime \prime} \mathrm{N} 101^{\circ} 20^{\prime} 33.5^{\prime \prime E}$ \\
\hline & CHA 001 & Wat Khao Tam Rat & $13^{\circ} 23^{\prime} 30.3^{\prime \prime} \mathrm{N} 101^{\circ} 44^{\prime} 39.6^{\prime \prime E}$ \\
\hline \multirow[t]{16}{*}{ Srakeo } & SRA 001 & Wat Tam Khao Chakan & $13^{\circ} 39^{\prime} 38.0^{\prime \prime} \mathrm{N} 102^{\circ} 05^{\prime} 02.8^{\prime \prime} \mathrm{E}$ \\
\hline & SRA 002 & Samnaksong Phuming & $13^{\circ} 38^{\prime} 52.5^{\prime \prime} \mathrm{N} 102^{\circ} 05^{\prime} 52.4^{\prime \prime E}$ \\
\hline & SRA 003 & Wat Tam Khaochan & $13^{\circ} 34^{\prime} 43.2^{\prime \prime} \mathrm{N} 102^{\circ} 05^{\prime} 34.8^{\prime \prime E}$ \\
\hline & SRA 004 & Wat Petpananikom & $13^{\circ} 29^{\prime} 17.9^{\prime \prime} \mathrm{N} 102^{\circ} 04^{\prime} 48.3^{\prime \prime E}$ \\
\hline & SRA 005 & Wat Khao Singto & $13^{\circ} 59^{\prime} 22.4^{\prime \prime} \mathrm{N} 102^{\circ} 00^{\prime} 29.3^{\prime \prime E}$ \\
\hline & SRA 006 & Wat Tam Maka & $13^{\circ} 47^{\prime} 49.6^{\prime \prime} \mathrm{N} 101^{\circ} 57^{\prime} 54.3^{\prime \prime} \mathrm{E}$ \\
\hline & SRA 007 & Wat Khao Sampung & $13^{\circ} 39^{\prime} 07.2^{\prime \prime} \mathrm{N} 101^{\circ} 56^{\prime} 53.5^{\prime \prime E}$ \\
\hline & SRA 008 & Wat Saitong & $13^{\circ} 32^{\prime} 48.1^{\prime \prime} \mathrm{N} 101^{\circ} 57^{\prime} 17.4^{\prime \prime} \mathrm{E}$ \\
\hline & SRA 009 & Khao Phaphueng & $13^{\circ} 28^{\prime} 27.1^{\prime \prime} \mathrm{N} 102^{\circ} 17^{\prime} 00.9^{\prime \prime} \mathrm{E}$ \\
\hline & SRA 010 & Khao Saraphee & $13^{\circ} 28^{\prime} 36.8^{\prime \prime} \mathrm{N} 102^{\circ} 19^{\prime} 06.3^{\prime \prime} \mathrm{E}$ \\
\hline & SRA 011 & Khao Laeum & $13^{\circ} 24^{\prime} 23.3^{\prime \prime} \mathrm{N} 102^{\circ} 18^{\prime} 05.3^{\prime \prime} \mathrm{E}$ \\
\hline & SRA 012 & Wat Tam Khao Phuheep & $13^{\circ} 23^{\prime} 12.5^{\prime \prime} \mathrm{N} 102^{\circ} 15^{\prime} 13.0^{\prime \prime} \mathrm{E}$ \\
\hline & SRA 013 & Wat Sabthavorn & $13^{\circ} 24^{\prime} 19.9^{\prime \prime} \mathrm{N} 102^{\circ} 16^{\prime} 29.6^{\prime \prime} \mathrm{E}$ \\
\hline & SRA 014 & Tam Pet Phoe Tong & $13^{\circ} 24^{\prime} 49.2^{\prime \prime} \mathrm{N} 102^{\circ} 19^{\prime} 31.3^{\prime \prime E}$ \\
\hline & SRA 015 & Tam Nam Pra Shiva & $13^{\circ} 19^{\prime} 15.2^{\prime \prime} \mathrm{N} 102^{\circ} 19^{\prime} 40.1^{\prime \prime E}$ \\
\hline & SRA 016 & Tam Saengtien & $13^{\circ} 18^{\prime} 57.2^{\prime \prime} \mathrm{N} 102^{\circ} 19^{\prime} 57.2^{\prime \prime E}$ \\
\hline \multirow[t]{2}{*}{ Pracheenburi } & PRA 001 & Wat Khao Teppitak & $13^{\circ} 39^{\prime} 37.4^{\prime \prime} \mathrm{N} 101^{\circ} 54^{\prime} 52.5^{\prime \prime E}$ \\
\hline & PRA 002 & Khao Tam Namtip & $13^{\circ} 39^{\prime} 19.2^{\prime \prime} \mathrm{N} 101^{\circ} 55^{\prime} 44.1^{\prime \prime} \mathrm{E}$ \\
\hline
\end{tabular}

\section{Taxon treatments}

\section{Diplommatina (crispata) khaochamaoensis Panha et al., 1998}

\section{Materials}

a. ScientificName: Diplommatina crispata khaochamaoensis; country: Thailand; stateProvince: Rayong; locality: Khao Pratun, Khaochamao District; verbatimCoordinates: $13^{\circ} 07^{\prime} 36.9 " \mathrm{~N} 101^{\circ} 35^{\prime} 56.6 " \mathrm{E}$; georeferenceProtocol: GPS; samplingProtocol: hand collecting; eventDate: 2004; habitat: limestone hills; individualCount: 8; lifeStage: adult; preparations: dry shell material; catalogNumber: ZRCBUU0019; recordedBy: Pongrat Dumrongrojwattana; identifiedBy: Pongrat Dumrongrojwattana; dateldentified: 2004; language: en; collectionCode: Mollusc; basisOfRecord: PreservedSpecimen

b. ScientificName: Diplommatina crispata khaochamaoensis; country: Thailand; stateProvince: Rayong; locality: Khao Pratun, Khaochamao District; verbatimCoordinates: 
$13^{\circ} 07^{\prime} 36.9^{\prime \prime} \mathrm{N} 101^{\circ} 35^{\prime} 56.6^{\prime \prime E}$; georeferenceProtocol: GPS; samplingProtocol: hand collecting; eventDate: 2004; habitat: limestone hills; individualCount: 16; lifeStage: adult; preparations: dry shell material; catalogNumber: ZRCBUU0029; recordedBy: Pongrat Dumrongrojwattana; identifiedBy: Pongrat Dumrongrojwattana; dateldentified: 2004; language: en; collectionCode: Mollusc; basisOfRecord: PreservedSpecimen

c. scientificName: Diplommatina crispata khaochamaoensis; country: Thailand; stateProvince: Chonburi; locality: Lublae Cave, Bothong District, Chonburi province; verbatimCoordinates: $13^{\circ} 09^{\prime} 09.0^{\prime \prime} \mathrm{N} 101^{\circ} 35^{\prime} 52.1 " \mathrm{E}$; georeferenceProtocol: GPS; samplingProtocol: hand collecting; eventDate: 2004; habitat: limestone hills; individualCount: 15; lifeStage: adult; preparations: dry shell material; catalogNumber: ZRCBUU0042; recordedBy: Pongrat Dumrongrojwattana; identifiedBy: Pongrat Dumrongrojwattana; dateldentified: 2004; language: en; collectionCode: Mollusc; basisOfRecord: PreservedSpecimen

d. scientificName: Diplommatina crispata khaochamaoensis; country: Thailand; stateProvince: Rayong; locality: Wat Tam Wattana Mongkol, Khaochamao District; verbatimCoordinates: $13^{\circ} 05^{\prime} 49.4^{\prime \prime} \mathrm{N} 101^{\circ} 36^{\prime} 25.1 " \mathrm{E}$; georeferenceProtocol: GPS; samplingProtocol: hand collecting; eventDate: 2004; habitat: limestone hills; individualCount: 16; lifeStage: adult; preparations: dry shell material; catalogNumber: ZRCBUU0047; recordedBy: Pongrat Dumrongrojwattana; identifiedBy: Pongrat Dumrongrojwattana; dateldentified: 2004; language: en; collectionCode: Mollusc; basisOfRecord: PreservedSpecimen

e. $\quad$ scientificName: Diplommatina crispata khaochamaoensis; country: Thailand; stateProvince: Chonburi; locality: Lublae Cave, Bothong District; verbatimCoordinates: $13^{\circ} 09^{\prime} 09.0^{\prime N} \mathrm{~N} 101^{\circ} 35^{\prime} 52.1 " \mathrm{E}$; georeferenceProtocol: GPS; samplingProtocol: hand collecting; eventDate: 2008; habitat: limestone hills; individualCount: 10; lifeStage: adult; preparations: dry shell material; catalogNumber: ZRCBUU0051; recordedBy: Pongrat Dumrongrojwattana; identifiedBy: Pongrat Dumrongrojwattana; dateldentified: 2008; language: en; collectionCode: Mollusc; basisOfRecord: PreservedSpecimen

f. scientificName: Diplommatina crispata khaochamaoensis; country: Thailand; stateProvince: Rayong; locality: Khao Pratun, Khaochamao District; verbatimCoordinates: $13^{\circ} 07^{\prime} 36.9^{\prime \prime} \mathrm{N} 101^{\circ} 35^{\prime} 56.6^{\prime \prime E}$; georeferenceProtocol: GPS; samplingProtocol: hand collecting; eventDate: 2008; habitat: limestone hills; individualCount: 10; lifeStage: adult; preparations: dry shell material; catalogNumber: ZRCBUU0079; recordedBy: Pongrat Dumrongrojwattana; identifiedBy: Pongrat Dumrongrojwattana; dateldentified: 2008; language: en; collectionCode: Mollusc; basisOfRecord: PreservedSpecimen

g. scientificName: Diplommatina crispata khaochamaoensis; country: Thailand; stateProvince: Chonburi; locality: Lublae Cave, Bothong District; verbatimCoordinates: 1309'09.0"N 101 $35^{\circ} 52.1^{\prime \prime} \mathrm{E}$; georeferenceProtocol: GPS; samplingProtocol: hand collecting; eventDate: 2015; habitat: limestone hills; individualCount: 5; lifeStage: adult; preparations: dry shell material; catalogNumber: ZRCBUU0086; recordedBy: Pongrat Dumrongrojwattana; identifiedBy: Pongrat Dumrongrojwattana; dateldentified: 2015; language: en; collectionCode: Mollusc; basisOfRecord: PreservedSpecimen

h. scientificName: Diplommatina crispata khaochamaoensis; country: Thailand; stateProvince: Rayong; locality: Wat Rattanatrikoon, Khaochamao District; verbatimCoordinates: $12^{\circ} 56^{\prime} 53.1^{\prime \prime} \mathrm{N} 101^{\circ} 46^{\prime} 37.1 " \mathrm{E}$; georeferenceProtocol: GPS; samplingProtocol: hand collecting; eventDate: 2013; habitat: limestone hills; individualCount: 24; lifeStage: adult; preparations: dry shell material; catalogNumber: ZRCBUU0098; recordedBy: Pongrat Dumrongrojwattana; identifiedBy: Pongrat Dumrongrojwattana; dateldentified: 2013; language: en; collectionCode: Mollusc; basisOfRecord: PreservedSpecimen 
i. scientificName: Diplommatina crispata khaochamaoensis; country: Thailand; stateProvince: Rayong; locality: Khao Pratun, Khaochamao District; verbatimCoordinates: $13^{\circ} 07^{\prime} 36.9^{\prime \prime} \mathrm{N} 101^{\circ} 35^{\prime} 56.6 " \mathrm{E}$; georeferenceProtocol: GPS; samplingProtocol: hand collecting; eventDate: 2015; habitat: limestone hills; individualCount: 18; lifeStage: adult; preparations: dry shell material; catalogNumber: ZRCBUU0107; recordedBy: Pongrat Dumrongrojwattana; identifiedBy: Pongrat Dumrongrojwattana; dateldentified: 2015; language: en; collectionCode: Mollusc; basisOfRecord: PreservedSpecimen

j. $\quad$ scientificName: Diplommatina crispata khaochamaoensis; country: Thailand; stateProvince: Chonburi; locality: Lublae Cave, Bothong District; verbatimCoordinates: $13^{\circ} 09^{\prime} 09.0^{\prime \prime} \mathrm{N} 101^{\circ} 35^{\prime} 52.1^{\prime \prime E}$; georeferenceProtocol: GPS; samplingProtocol: hand collecting; eventDate: 2015; habitat: limestone hills; individualCount: 5; lifeStage: adult; preparations: dry shell material; catalogNumber: ZRCBUU0108; recordedBy: Pongrat Dumrongrojwattana; identifiedBy: Pongrat Dumrongrojwattana; dateldentified: 2015; language: en; collectionCode: Mollusc; basisOfRecord: PreservedSpecimen

k. scientificName: Diplommatina crispata khaochamaoensis; country: Thailand; stateProvince: Rayong; locality: Khao Pratun, Khaochamao District; verbatimCoordinates: $13^{\circ} 07^{\prime} 36.9^{\prime \prime} \mathrm{N} 101^{\circ} 35^{\prime} 56.6^{\prime \prime E}$; georeferenceProtocol: GPS; samplingProtocol: hand collecting; eventDate: 2015; habitat: limestone hills; individualCount: 5; lifeStage: adult; preparations: dry shell material; catalogNumber: ZRCBUU0109; recordedBy: Pongrat Dumrongrojwattana; identifiedBy: Pongrat Dumrongrojwattana; dateldentified: 2015; language: en; collectionCode: Mollusc; basisOfRecord: PreservedSpecimen

I. scientificName: Diplommatina crispata khaochamaoensis; country: Thailand; stateProvince: Rayong; locality: Khao Pratun, Khaochamao District; verbatimCoordinates: $13^{\circ} 07^{\prime} 36.9^{\prime N} \mathrm{~N} 101^{\circ} 35^{\prime} 56.6^{\prime \prime} \mathrm{E}$; georeferenceProtocol: GPS; samplingProtocol: hand collecting; eventDate: 2018; habitat: limestone hills; individualCount: 22; lifeStage: adult; preparations: dry shell material; catalogNumber: ZRCBUU0534; recordedBy: Pongrat Dumrongrojwattana; identifiedBy: Pongrat Dumrongrojwattana; dateldentified: 2018; language: en; collectionCode: Mollusc; basisOfRecord: PreservedSpecimen

m. scientificName: Diplommatina crispata khaochamaoensis; country: Thailand; stateProvince: Chonburi; locality: Lublae Cave, Bothong District; verbatimCoordinates: $13^{\circ} 09^{\prime} 09.0^{\prime \prime} \mathrm{N} 101^{\circ} 35^{\prime} 52.1^{\prime \prime E}$; georeferenceProtocol: GPS; samplingProtocol: hand collecting; eventDate: 2019; habitat: limestone hills; individualCount: 22; lifeStage: adult; preparations: dry shell material; catalog Number: ZRCBUU0619; recordedBy: Pongrat Dumrongrojwattana; identifiedBy: Pongrat Dumrongrojwattana; dateldentified: 2019; language: en; collectionCode: Mollusc; basisOfRecord: PreservedSpecimen

n. $\quad$ scientificName: Diplommatina crispata khaochamaoensis; country: Thailand; stateProvince: Rayong; locality: Wat Tam Wattana Mongkol, Khaochamao District; verbatimCoordinates: $13^{\circ} 05^{\prime} 49.4^{\prime \prime} \mathrm{N} 101^{\circ} 36^{\prime} 25.1 " \mathrm{E}$; georeferenceProtocol: GPS; samplingProtocol: hand collecting; eventDate: 2020; habitat: limestone hills; individualCount: 11; lifeStage: adult; preparations: dry shell material; catalogNumber: ZRCBUU0739; recordedBy: Pongrat Dumrongrojwattana; identifiedBy: Pongrat Dumrongrojwattana; dateldentified: 2020; language: en; collectionCode: Mollusc; basisOfRecord: PreservedSpecimen

o. $\quad$ scientificName: Diplommatina crispata khaochamaoensis; country: Thailand; stateProvince: Rayong; locality: Pradoo Cave, Khaochamao District; verbatimCoordinates: $13^{\circ} 05^{\prime} 40.8^{\prime \prime} \mathrm{N} 101^{\circ} 36^{\prime} 25.1 " \mathrm{E}$; georeferenceProtocol: GPS; samplingProtocol: hand collecting; eventDate: 2020; habitat: limestone hills; individualCount: 11; lifeStage: adult; preparations: dry shell material; catalogNumber: ZRCBUU0740; recordedBy: Piyaporn Muenrit; identifiedBy: Pongrat Dumrongrojwattana; 
dateldentified: 2020; language: en; collectionCode: Mollusc; basisOfRecord:

PreservedSpecimen

p. $\quad$ scientificName: Diplommatina crispata khaochamaoensis; country: Thailand; stateProvince: Rayong; locality: Khao Pratun, Khaochamao District; verbatimCoordinates: $13^{\circ} 07^{\prime} 36.9^{\prime \prime N} 101^{\circ} 35^{\prime} 56.6^{\prime \prime E}$; georeferenceProtocol: GPS; samplingProtocol: hand collecting; eventDate: 2020; habitat: limestone hills; individualCount: 11; lifeStage: adult; preparations: dry shell material; catalogNumber: ZRCBUU0741; recordedBy: Sasicha Techama; identifiedBy: Pongrat Dumrongrojwattana; dateldentified: 2020; language: en; collectionCode: Mollusc; basisOfRecord: PreservedSpecimen

q. $\quad$ scientificName: Diplommatina crispata khaochamaoensis; country: Thailand; stateProvince: Rayong; locality: Khao Pratun, Khaochamao District; verbatimCoordinates: $13^{\circ} 07^{\prime} 36.9^{\prime \prime} \mathrm{N} 101^{\circ} 35^{\prime} 56.6^{\prime \prime} \mathrm{E}$; georeferenceProtocol: GPS; samplingProtocol: hand collecting; eventDate: 2020; habitat: limestone hills; individualCount: 11; lifeStage: adult; preparations: dry shell material; catalogNumber: ZRCBUU0742; recordedBy: Onchira Saenkamon; identifiedBy: Pongrat Dumrongrojwattana; dateldentified: 2020; language: en; collectionCode: Mollusc; basisOfRecord: PreservedSpecimen

r. scientificName: Diplommatina crispata khaochamaoensis; country: Thailand; stateProvince: Rayong; locality: Khao Pratun, Khaochamao District; verbatimCoordinates: $13^{\circ} 07^{\prime} 36.9^{\prime \prime N} 11^{\circ} 35^{\prime} 56.6^{\prime \prime} \mathrm{E}$; georeferenceProtocol: GPS; samplingProtocol: hand collecting; eventDate: 2020; habitat: limestone hills; individualCount: 24; lifeStage: adult; preparations: dry shell material; catalogNumber: ZRCBUU0744; recordedBy: Sirilak BuOn; identifiedBy: Pongrat Dumrongrojwattana; dateldentified: 2020; language: en; collectionCode: Mollusc; basisOfRecord: PreservedSpecimen

s. $\quad$ scientificName: Diplommatina crispata khaochamaoensis; country: Thailand; stateProvince: Rayong; locality: Khao Pratun, Khaochamao District; verbatimCoordinates: $13^{\circ} 07^{\prime} 36.9^{\prime \prime} \mathrm{N} 101^{\circ} 35^{\prime} 56.6^{\prime \prime E}$; georeferenceProtocol: GPS; samplingProtocol: hand collecting; eventDate: 2020; habitat: limestone hills; individualCount: 11; lifeStage: adult; preparations: dry shell material; catalogNumber: ZRCBUU0758; recordedBy: Noppadon Wiboonpuech; identifiedBy: Pongrat Dumrongrojwattana; dateldentified: 2020; language: en; collectionCode: Mollusc; basisOfRecord: PreservedSpecimen

\section{Distribution}

Limestone areas in Chonburi and Rayong provinces

\section{Diplommatina burapha Dumrongrojwattana, Kamtuptim \& Wongkamhaeng} 2020, sp. n.

\section{- ZooBank B98B3418-9B9F-4F5E-AD09-C6DB5AEBE2AA}

\section{Nomenclature}

We named this new species "burapha," which means "eastern." This refers to the type locality, which is located in the east and is also in honour of Burapha University, the workplace of the authors. 


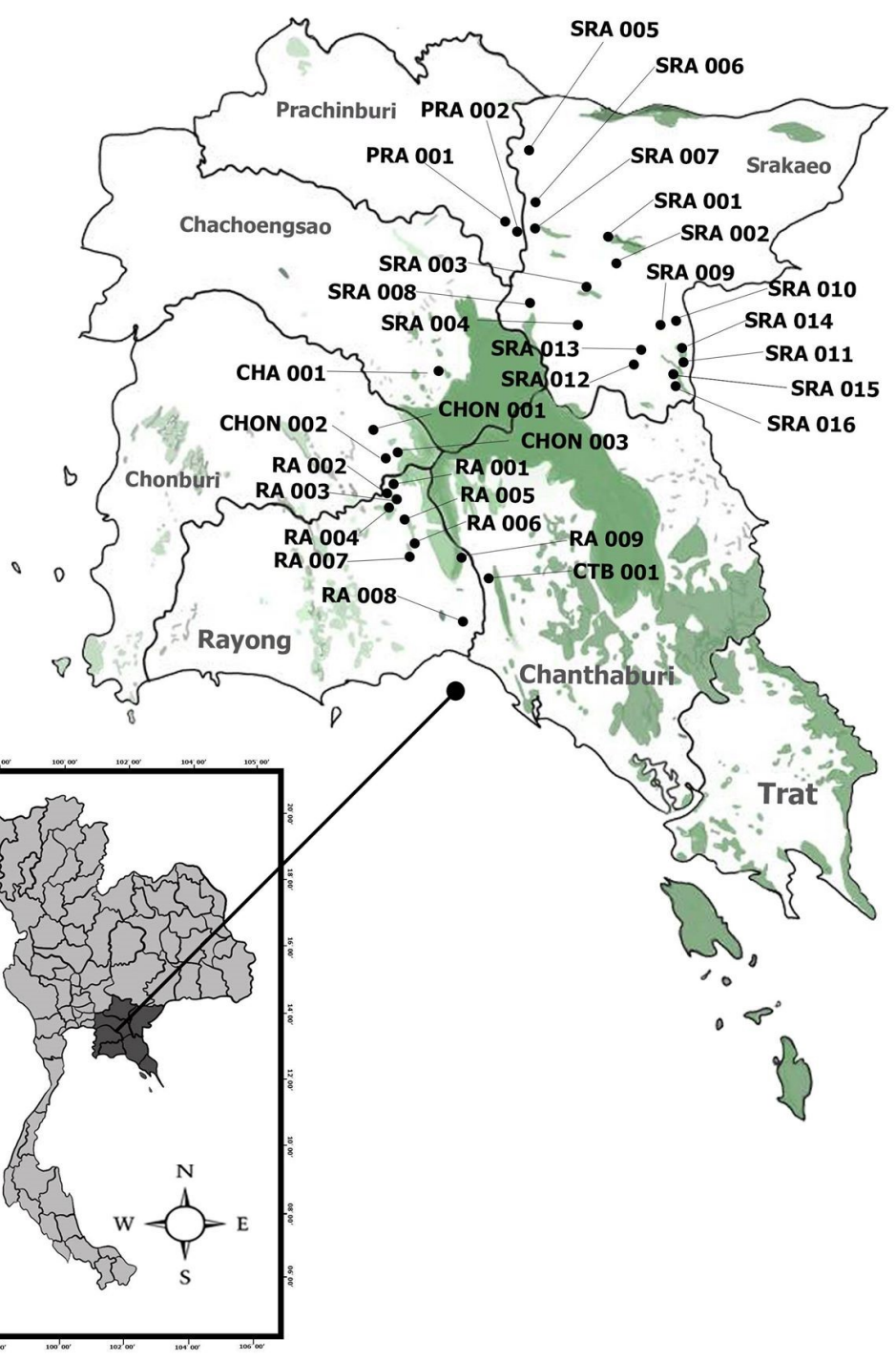

Figure 1. doi

Collecting sites. The code indicated in the map follows the list in Table 1. 


\section{Materials}

\section{Holotype:}

a. $\quad$ scientificName: Diplommatina burapha; country: Thailand; stateProvince: Srakeo; locality: Wat Tam Khaochan, Khaochakan District; verbatimElevation: 120-130 m; verbatimCoordinates: $13^{\circ} 34^{\prime} 43.2^{\prime \prime} \mathrm{N} 102^{\circ} 05^{\prime} 34.8^{\prime \prime} \mathrm{E}$; decimalLatitude: 13.525939 ; decimalLongitude: 102.076315; georeferenceProtocol: GPS; samplingProtocol: hand collecting; eventDate: 2020; habitat: The wall of limestone hills that is located in a temple which is surrounded by variety of plants including the member of Euphorbiaceae, Leguminosae and Orchidaceae.; individualCount: 1; lifeStage: adult; preparations: dry shell material; catalogNumber: ZRCBUU-0747; recordedBy: Pongrat Dumrongrojwattana; disposition: in collection; identifiedBy: Pongrat Dumrongrojwattana; dateldentified: 2020; language: en; collectionCode: Mollusc; basisOfRecord: PreservedSpecimen

\section{Paratype:}

a. $\quad$ scientificName: Diplommatina burapha; country: Thailand; stateProvince: Srakeo; locality: Wat Tam Khaochan, Khaochakan District; verbatimCoordinates: $13^{\circ} 34^{\prime} 43.2^{\prime \prime} \mathrm{N}$ $102^{\circ} 05^{\prime} 34.8 " \mathrm{E}$; georeferenceProtocol: GPS; samplingProtocol: hand collecting; eventDate: 2020; habitat: The wall of three limestone hills that is located in a temple which issurrounded by variety of plants including the members of Euphorbiaceae, Leguminosae and Orchidaceae.; individualCount: 26; lifeStage: adult; preparations: dry shell material; catalogNumber: ZRCBUU 0748; recordedBy: Pongrat Dumrongrojwattana; identifiedBy: Pongrat Dumrongrojwattana; dateldentified: 2020; language: en; collectionCode: Mollusc; basisOfRecord: PreservedSpecimen

\section{Description}

Holotype: Shell height $3.59 \mathrm{~mm}$, shell width $1.91 \mathrm{~mm}$. Aperture height $0.93 \mathrm{~mm}$, aperture width $1.86 \mathrm{~mm}$. Shell width/shell height ratio $=0.53$. Aperture width/aperture height ratio $=1.15$ (Fig. $2 \mathrm{~A})$.

Paratypes: Shell height 3.59-4.35 mm $(4.11 \pm 0.11 \mathrm{~mm})$ shell width 1.91-2.35 mm $(2.21 \pm 0.07 \mathrm{~mm})$. Aperture height 0.93-1.19 mm (1.08 $\pm 0.06 \mathrm{~mm})$. Aperture width $1.07-1.47 \mathrm{~mm}(1.39 \pm 0.07 \mathrm{~mm})$. Shell width/shell height ratio $=0.54 \pm 0.02$. Aperture width/aperture height ratio $=1.29 \pm 0.05$.

Shell minute dextral, elongated narrow, turreted with $73 / 4$ round whorls that increase regularly in size and diameter until the last whorl; protoconch orange, smooth, consisting of $1 \frac{1}{4}$ whorls, covered with minute pits; teleoconch light orange, consists of about $5 \frac{3}{4}$ whorls that increase regularly in size and diameter until the last whorl; spires shouldered; sculpture consists of rather even and rather widely-spaced radial ribs with about $8 \mathrm{ribs} / \mathrm{mm}$ on the penultimate whorl and about $6 \mathrm{ribs} / \mathrm{mm}$ on the body whorl, the discernible spiral striae between the ribspresent; sutures deep; umbilicus closed; aperture round; peristome is thickened and expanded and doubled; columellar lamella rather bluntly rounded (Fig. 4). 


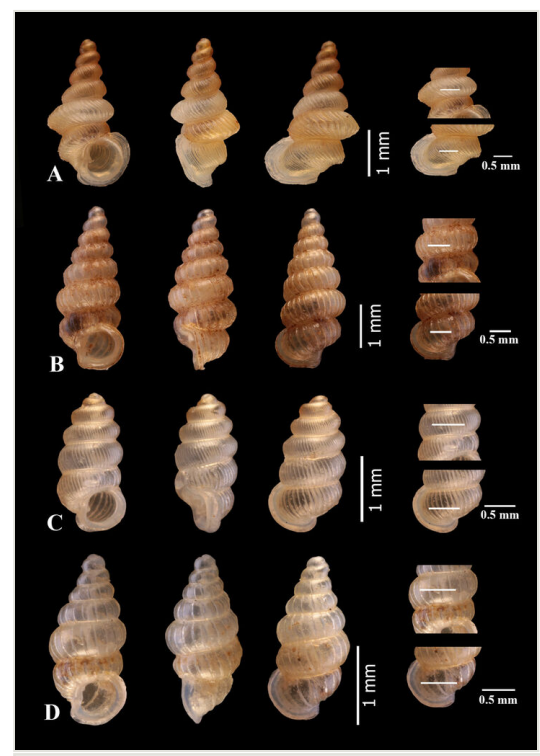

Figure 2. doi

Four Diplommatina species from eastern Thailand. A) Diplommatina crispata khaochamaoensis Panha et al., 1998, B) D. burapha sp. n., C) D. chadathongae sp. nov. and D) $D$. chantaburiensis $\mathrm{sp}$. $\mathrm{n}$. Each species shows a frontal view, lateral view, dorsal view and the number of ribs per $0.5 \mathrm{~mm}$ on the penultimate whorl and body whorl, respectively.

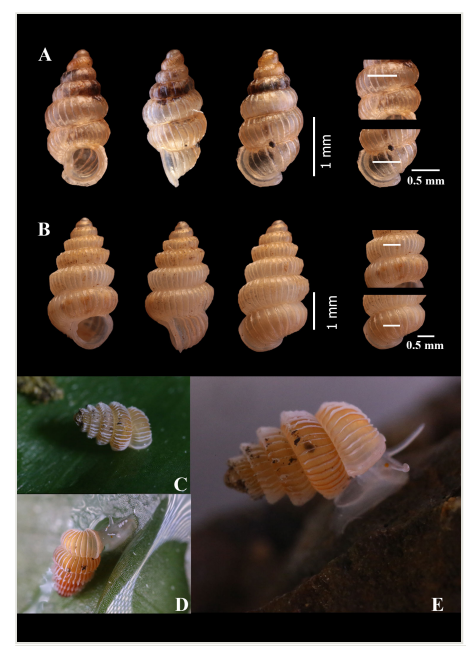

Figure 3. doi

Two Diplommatina species from eastern Thailand. A) D. fusiformis spec. nov., B-E) $D$. khwantongae spec. nov., B) Holotype, C-D) Living snail. (Photos by Ms. Rattanawadee Tekavong). Each species shows a frontal view, lateral view, dorsal view and the number of ribs per $0.5 \mathrm{~mm}$ on the penultimate whorl and body whorl, respectively. 


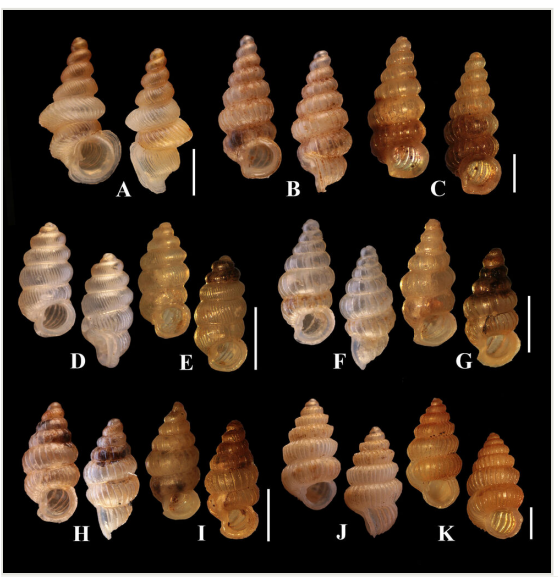

Figure 4. doi

Synopsis of eastern diplommatind species. A) Diplommatina crispata khaochamaoensis, B-C) Holotype and paratypes of $D$. burapha sp. n., D-E) Holotype and paratypes of $D$. chadathongae sp. n., F-G) Holotype and paratypes of $D$. chantaburiensis sp. $\mathrm{n}$., $\mathrm{H}-\mathrm{I}$ ) Holotype and paratypes of $D$. fusiformis $\mathrm{sp} . \mathrm{n}$. and J-K) Holotype and paratypes of $D$. khwantongae sp. n. Scale bar $=1 \mathrm{~mm}$.

\section{Diagnosis}

Shell minute, dextral, turreted, translucent, whitish; protoconch smooth; teleoconch sculpture of widely-spaced radial ribs with discernible spiral striae between the ribs, Aperture rounded, columellar lamella well developed, peristome doubled, thickened and expanded.

\section{Etymology}

We named this new species "burapha," which means "eastern." This refers to the type locality, which is located in the east and is also in honour of Burapha University, the workplace of the authors.

\section{Distribution}

This species is known only from the type locality.

\section{Taxon discussion}

The size of the shell, shape of the shell and protoconch of $D$. burapha sp. n. are similar to D. doichiangdao Panha \& Burch, 1998 from Doi Chiang Dao, northern Thailand and Laotian species, $D$. belonis Möllendorff, 1900 . The spire of $D$. burapha sp. n. is relatively broader than that of those two species. The enlarged penultimate whorl and the body whorl of $D$. burapha sp. $\mathrm{n}$. are relatively similar in diameter, while the last whorls of both $D$. doichiangdao and $D$. belonis are smaller in diameter than the 
enlarged penultimate whorl. This new species columellar is poorly developed, while well developed in $D$. doichiangdao and $D$. belonis.

\section{Diplommatina chadathongae Kamtuptim, Dumrongrojwattana \& Wongkamhaeng 2020, sp. $n$.}

- ZooBank 4ACB0FCD-80A2-44C1-A213-3734A0F3B5F0

\section{Materials}

Holotype:

a. ScientificName: Diplommatina chadathongae; country: Thailand; stateProvince: Chanthaburi; locality: Wat Khaowongkotrujiwongsaram, Kanghaengmaeo District; verbatimCoordinates: $12^{\circ} 53^{\prime} 11.4^{\prime \prime} \mathrm{N} 101^{\circ} 49^{\prime} 06.2^{\prime \prime E}$; georeferenceProtocol: GPS; samplingProtocol: hand collecting; eventDate: 2020; habitat: limestone hills; individualCount: 1; lifeStage: adult; preparations: dry shell material; catalogNumber: ZRCBUU-0092; recordedBy: Pongrat Dumrongrojwattana; identifiedBy: Pongrat Dumrongrojwattana; dateldentified: 2012; language: en; collectionCode: Mollusc; basisOfRecord: PreservedSpecimen

\section{Paratype:}

a. ScientificName: Diplommatina chadathongae; country: Thailand; stateProvince: Chanthaburi; locality: Wat Tam Khaochan, Khaochakan District; verbatimCoordinates: $12^{\circ} 53^{\prime} 11.4^{\prime \prime} \mathrm{N} 101^{\circ} 49^{\prime} 06.2^{\prime \prime E}$; georeferenceProtocol: GPS; samplingProtocol: hand collecting; eventDate: 2020; habitat: limestone hills; individualCount: 29; lifeStage: adult; preparations: dry shell material; catalogNumber: ZRCBUU-0093; recordedBy: Pongrat Dumrongrojwattana; identifiedBy: Pongrat Dumrongrojwattana; dateldentified: 2012; language: en; collectionCode: Mollusc; basisOfRecord: PreservedSpecimen

\section{Description}

Holotype. Shell height $1.61 \mathrm{~mm}$, shell width $0.78 \mathrm{~mm}$. Aperture height $0.50 \mathrm{~mm}$, aperture width $0.60 \mathrm{~mm}$. Shell width/shell height ratio $=0.49$. Aperture width/aperture height ratio $=1.21$ (Fig. $2 \mathrm{C}$ ).

Paratypes (6 shells). Shell height $1.93-2.07 \mathrm{~mm}(1.98 \pm 0.05 \mathrm{~mm})$, shell width 0.84-0.93 mm $(0.88 \pm 0.03 \mathrm{~mm})$. Aperture height $0.51-0.63 \mathrm{~mm}(0.58 \pm 0.05 \mathrm{~mm})$. Aperture width $0.59-0.67 \mathrm{~mm}(0.63 \pm 0.02 \mathrm{~mm})$. Shell width/shell height ratio $=0.45 \pm$ 0.01 . Aperture width/aperture height ratio $=1.09 \pm 0.07$.

Shell minute dextral, cylindrical, translucent, whitish, with about $5 \frac{3 / 4}{4}$ whorls that increase regularly in size and diameter until the last whorl; protoconch orange, smooth, consisting of $1 \frac{1}{2}$ whorls; teleoconch light orange, consisting of about $4 \frac{1 / 4}{4}$ keeled whorls; sculpture consists of strong, rather narrowly-spaced radial ribs which are about $20 \mathrm{ribs} / \mathrm{mm}$ on the penultimate whorl and about $14 \mathrm{ribs} / \mathrm{mm}$ on the body whorl; sutures well impressed; umbilicus closed; aperture round; peristome thickened and expanded and doubled; columellar lamella well developed and directed anteriorly (Fig. 4). 


\section{Diagnosis}

Shell minute, dextral, cylindrical, translucent, whitish, teleoconch sculpture of narrowlyspaced axial ribs, whorls aperture rounded, columellar lamella well developed, peristome thickened, expanded and doubled.

\section{Etymology}

We named this new species "chadathongae" in memory of the second author's beloved mother, Ms. Wiang Chadathong.

\section{Distribution}

Only known from the type locality.

\section{Taxon discussion}

Diplommatina chadathongae sp. $\mathrm{n}$. is another one of the Thai smallest Diplommatina species (average shell height $=1.69 \pm 0.04 \mathrm{~mm}$ ) and same size as $D$. krabiensis Panha \& Burch, 1998 (shell height of type specimens are 1.6-1.7 mm and average shell height $=1.69 \pm 0.04 \mathrm{~mm}$ ). This new species differs from $D$. krabiensis by its dextral, cylindrical, narrowly-fine radial ribs, while the smallest species is sinistral, shell fusiform or ovate with widely-spaced radial ribs. This new species differs from Diplommatina miriensis Godwin-Austen, 1917 from Nepal by its shell being more slender and columellar lamella well developed.

\section{Diplommatina chantaburiensis Dumrongrojwattana, Kamtuptim \& Wongkamhaeng 2020, sp. n.}

- ZooBank 2573D215-407A-42C5-AD42-7E365477CA7D

\section{Materials}

Holotype:

a. $\quad$ scientificName: Diplommatina chantaburiensis; country: Thailand; stateProvince: Chanthaburi; locality: Wat Khao Wong Kot, Kanghaengmaeo District; verbatimCoordinates: $12^{\circ} 52^{\prime} 47.3^{\prime \prime} \mathrm{N} 101^{\circ} 49^{\prime} 13.8^{\prime \prime E}$; georeferenceProtocol: GPS; samplingProtocol: hand collecting; eventDate: 2020; habitat: limestone hills; individualCount: 1; lifeStage: adult; preparations: dry shell material; catalogNumber: ZRCBUU-0090; recordedBy: Pongrat Dumrongrojwattana; identifiedBy: Pongrat Dumrongrojwattana; dateldentified: 2012; language: en; collectionCode: Mollusc; basisOfRecord: PreservedSpecimen

\section{Paratype:}

a. scientificName: Diplommatina chantaburiensis; country: Thailand; stateProvince: Chanthaburi; locality: Wat Khao Wong Kot, Kanghaengmaeo District; verbatimCoordinates: $12^{\circ} 52^{\prime} 47.3^{\prime \prime} \mathrm{N} 101^{\circ} 49^{\prime} 13.8^{\prime \prime} \mathrm{E}$; georeferenceProtocol: GPS; samplingProtocol: hand collecting; eventDate: 2020; habitat: limestone hills; 
individualCount: 29; lifeStage: adult; preparations: dry shell material; catalogNumber: ZRCBUU-0091; recordedBy: Pongrat Dumrongrojwattana; identifiedBy: Pongrat Dumrongrojwattana; dateldentified: 2012; language: en; collectionCode: Mollusc; basisOfRecord: PreservedSpecimen

\section{Description}

Holotype. Shell height $3.56 \mathrm{~mm}$, shell width $1.64 \mathrm{~mm}$. Aperture height $1.03 \mathrm{~mm}$, aperture width $1.23 \mathrm{~mm}$. Shell width/shell height ratio $=0.46$. Aperture width/aperture height ratio $=1.19$ (Fig. $2 \mathrm{D})$.

Paratypes (7 shells). Shell height $2.56-2.85 \mathrm{~mm}(2.67 \pm 0.10 \mathrm{~mm})$ shell width $1.13-1.28 \mathrm{~mm}(1.24 \pm 0.05 \mathrm{~mm})$. Aperture height $0.73-0.85 \mathrm{~mm}(0.77 \pm 0.06 \mathrm{~mm})$. Aperture width $0.73-1.01 \mathrm{~mm}(0.93 \pm 0.08 \mathrm{~mm})$. Shell width/shell height ratio $=0.46 \pm$ 0.02. Aperture width/aperture height ratio $=1.20 \pm 0.13$.Shell minute dextral, fusiform, translucent, whitish, with about 7 whorls that increase regularly in both size and diameter until the last whorl which is slightly smaller in diameter than the penultimate whorl; protoconch large, whitish, smooth, surface cover with minute pits, consisting of $1 \frac{1}{4}$ whorls; teleoconch about $5 \frac{1 / 4}{4}$ whorls, whitish; sculpture consists of thin, widelyspaced radial ribs which about 9 ribs $/ \mathrm{mm}$ on the penultimate whorl and about 6 ribs/ $\mathrm{mm}$ on the body whorl, discernible spiral striae between the ribs absent; sutures deeply impressed; umbilicus closed; aperture rounded; peristome thicked and expanded; columellar lamella is relatively small (Fig. 4).

\section{Diagnosis}

Shell minute, dextral, translucent, whitish, protoconch large, teleoconch sculpture consisting of widely-spaced axial ribs, spiral striae between the ribs absent; aperture rounded, columellar lamella relatively small, peristome thickened and expanded.

\section{Etymology}

The species name "chantaburiensis" refers to Chantaburi, the Province of the type locality.

\section{Distribution}

Only known from the type locality.

\section{Taxon discussion}

Diplommatina chantaburiensis sp. $\mathrm{n}$. is very similar to $D$. fusiformis $\mathrm{sp}$. $\mathrm{n}$. Nevertheless, $D$. chantaburiensis $\mathrm{sp}$. $\mathrm{n}$. has larger protoconch, shell more slender and no spiral striae between the radial ribs. This new species is also similar to the southern species $D$. hidagai Panha, 1998, but differs by its prominent protoconch, more widely-radial ribs and columellar lamella is poorly developed. 


\section{Diplommatina fusiformis Dumrongrojwattana, Kamtuptim \& Wongkamhaeng 2020, sp. n.}

\section{- ZooBank 92F4C2C9-E2CC-43EA-825A-5052282885ED}

\section{Materials}

Holotype:

a. ScientificName: Diplommatina fusiformis; country: Thailand; stateProvince: Rayong; locality: Wat Rattanatrikoon, Khaochamao District; verbatimCoordinates: $12^{\circ} 56^{\prime} 53.1^{\prime \prime} \mathrm{N}$ $101^{\circ} 46$ '37.1"E; georeferenceProtocol: GPS; samplingProtocol: hand collecting; eventDate: 2020; habitat: limestone hills; individualCount: 1; lifeStage: adult; preparations: dry shell material; catalogNumber: ZRCBUU-0105; recordedBy: Pongrat Dumrongrojwattana; identifiedBy: Pongrat Dumrongrojwattana; dateldentified: 2015; language: en; collectionCode: Mollusc; basisOfRecord: PreservedSpecimen

Paratype:

a. $\quad$ scientificName: Diplommatina fusiformis; country: Thailand; stateProvince: Rayong; locality: Wat Rattanatrikoon, Khaochamao District; verbatimCoordinates: $12^{\circ} 56^{\prime} 53.1 " \mathrm{~N}$ $101^{\circ} 46$ '37.1"E; georeferenceProtocol: GPS; samplingProtocol: hand collecting; eventDate: 2020; habitat: limestone hills; individualCount: 18; lifeStage: adult; preparations: dry shell material; catalogNumber: ZRCBUU-0104; recordedBy: Pongrat Dumrongrojwattana; identifiedBy: Pongrat Dumrongrojwattana; dateldentified: 2015; language: en; collectionCode: Mollusc; basisOfRecord: PreservedSpecimen

\section{Description}

Holotype. Shell height $2.66 \mathrm{~mm}$, shell width $1.22 \mathrm{~mm}$. Aperture height $0.76 \mathrm{~mm}$, aperture width $0.94 \mathrm{~mm}$. Shell width/shell height ratio $=0.46$. Aperture width/aperture height ratio $=1.23($ Fig. $3 \mathrm{~A})$.

Paratypes (5 shells). Shell height $2.66-2.74 \mathrm{~mm}(2.65 \pm 0.07 \mathrm{~mm})$ shell width $1.21-1.29 \mathrm{~mm}(1.25 \pm 0.03 \mathrm{~mm})$. Aperture height $0.76-0.87 \mathrm{~mm}(0.78 \pm 0.06 \mathrm{~mm})$. Aperture width $0.82-0.98 \mathrm{~mm}(0.91 \pm 0.06 \mathrm{~mm})$. Shell width/shell height ratio $=0.47 \pm$ 0.02 . Aperture width/aperture height ratio $=1.18 \pm 0.10$.

Description. Shell minute dextral, fusiform, translucent, light orange, with about $61 / 2$ whorls that increase regularly in size and diameter until the last whorl which is slightly smaller in diameter than the penultimate whorl; protoconch orange, smooth, consisting of $1 \frac{1 / 4}{4}$ whorls; teleoconch light orange, about $5 \frac{1}{4}$ whorls; sculpture consists of thin, widely-spaced radial ribs with discernible spiral striae between the ribs; there are about $10 \mathrm{ribs} / \mathrm{mm}$ on the penultimate whorl and about $8 \mathrm{ribs} / \mathrm{mm}$ on the body whorl; sutures deep; umbilicus closed; aperture rounded, peristome thickened and expanded; columellar lamella relatively small (Fig. 4) . 


\section{Diagnosis}

Diagnosis. Shell minute, dextral, translucent light orange, teleoconch sculpture consisting of widely-spaced axial ribs with spiral striae between the ribs; aperture rounded, columellar lamella relatively small, peristome, thickened and expanded.

\section{Etymology}

We named this new species "fusiformis" based on its fusiform shell shape.

\section{Distribution}

Only known from the type locality.

\section{Taxon discussion}

Diplommatina fusiformis sp. $\mathrm{n}$. is similar to the southern species, D. hidagai Panha, 1998, but it differs by its more widely-ribbed shell, its more slender spire and its less prominent columellar lamella.

\section{Diplommatina khwantongae Dumrongrojwattana, Kamtuptim \& Wongkamhaeng 2020, sp. n.}

\section{- ZooBank 4311F0B5-9CA7-460F-9637-1C11F9BEDD86}

\section{Materials}

Holotype:

a. $\quad$ scientificName: Diplommatina khwantongae; country: Thailand; stateProvince: Chonburi; locality: Lub Lae Cave, Bothong district, Chonburi Province; verbatimCoordinates: $13^{\circ} 09^{\prime} 09.9^{\prime \prime} \mathrm{N} 101^{\circ} 35^{\prime} 52.4^{\prime \prime E}$; georeferenceProtocol: GPS; samplingProtocol: hand collecting; eventDate: 2020; habitat: limestone hills; individualCount: 1; lifeStage: adult; preparations: dry shell material; catalogNumber: ZRCBUU-0190; recordedBy: Kanita Khwantong; identifiedBy: Kanita Khwantong; dateldentified: 2015; language: en; collectionCode: Mollusc; basisOfRecord: PreservedSpecimen

Paratypes:

a. $\quad$ scientificName: Diplommatina khwantongae; country: Thailand; stateProvince: Chonburi; locality: Lub Lae Cave, Bothong district, Chonburi Province; verbatimCoordinates: $13^{\circ} 09^{\prime} 09.9^{\prime \prime N} 101^{\circ} 35^{\prime} 52.4 " \mathrm{E}$; georeferenceProtocol: GPS; samplingProtocol: hand collecting; eventDate: 2020; habitat: limestone hills; individualCount: 5; lifeStage: adult; preparations: dry shell material; catalogNumber: ZRCBUU-0102; recordedBy: Kanita Khwantong; identifiedBy: Kanita Khwantong; dateldentified: 2015; language: en; collectionCode: Mollusc; basisOfRecord: PreservedSpecimen

b. $\quad$ scientificName: Diplommatina khwantongae; country: Thailand; stateProvince: Chonburi; locality: Lub Lae Cave, Bothong district, Chonburi Province; verbatimCoordinates: $13^{\circ} 09^{\prime} 09.9^{\prime \prime} \mathrm{N} 101^{\circ} 35^{\prime} 52.4^{\prime \prime E}$; georeferenceProtocol: GPS; samplingProtocol: hand collecting; eventDate: 2020; year: 2020; month: 7; day: 11; habitat: limestone hills; individualCount: 5; lifeStage: adult; preparations: dry shell material; catalogNumber: 
ZRCBUU-0757; recordedBy: Rattanawadee Tekavong; identifiedBy: Pongrat
Dumrongroiwattana; dateldentified: 2015; language: en; collectionCode: Mollusc;
basisOfRecord: PreservedSpecimen

Other material:

a. $\quad$ scientificName: Diplommatina khwantongae; country: Thailand; stateProvince: Chonburi; locality: Lub Lae Cave, Bothong district, Chonburi Province; verbatimCoordinates: $13^{\circ} 09^{\prime} 09.9^{\prime \prime} \mathrm{N} 101^{\circ} 35^{\prime} 52.4^{\prime \prime E}$; georeferenceProtocol: GPS; samplingProtocol: hand collecting; eventDate: 2020; year: 2020; month: 7; day: 11; habitat: limestone hills; individualCount: 3; lifeStage: adult; catalogNumber: ZRCBUU-0759; occurrenceRemarks: Three live specimens were collected and reared in the laboratory for few months. All specimens were preserved in ethanol.; recordedBy: Rattanawadee Tekavong; identifiedBy: Pongrat Dumrongroiwattana; dateldentified: 2015; language: en; collectionCode: Mollusc; basisOfRecord: PreservedSpecimen

\section{Description}

Holotype- Shell height $3.59 \mathrm{~mm}$, shell width $1.91 \mathrm{~mm}$. Aperture height $0.93 \mathrm{~mm}$, aperture width $1.86 \mathrm{~mm}$. Shell width/shell height ratio $=0.53$. Aperture width/aperture height ratio $=1.15$. (Fig. 3B-E).

Paratypes: (6 shells). Shell height $1.68-1.76 \mathrm{~mm}(1.69 \pm 0.04 \mathrm{~mm})$, shell width $0.78-0.81 \mathrm{~mm}(0.81 \pm 0.04 \mathrm{~mm})$. Aperture height $0.46-0.58 \mathrm{~mm}(0.53 \pm 0.04 \mathrm{~mm})$. Aperture width $0.53-0.61 \mathrm{~mm}(0.59 \pm 0.03 \mathrm{~mm})$. Shell width/shell height ratio $=0.48 \pm$ 0.03. Aperture width/aperture height ratio $=1.12 \pm 0.07$.

Shell minute dextral, cylindrical, light orange, with about 7 whorls that increase regularly in size and diameter until the last whorl; protoconch orange, smooth, consisting of $1 \frac{1 / 4}{4}$ whorls, covered with minute pits; teleoconch light orange, consisting of about $5 \frac{3}{4}$ whorls; spires shouldered; sculpture consists of rather even and widelyspaced thin radial ribs with discernible spiral striae between the ribs; there are about 8 $\mathrm{ribs} / \mathrm{mm}$ on the penultimate whorl and about $6 \mathrm{ribs} / \mathrm{mm}$ on the body whorl; sutures deep; umbilicus closed; aperture round; peristome thickened and expanded and doubled; columellar lamella rather bluntly rounded.

Animal. Living animals had a greyish body, head, tentacles and foot, with an orange eye located at the base of each tentacle (Fig. 3C-E). The operculum was round and corneous. Snails were found living in leaf litter and plant debris in the limestone hills. Due to the small population of each species, the live specimens of all species found in this study are hard to find. More observation and further study on anatomy are needed.

\section{Diagnosis}

Shell minute, dextral, cylindrical, light orange, teleoconch sculpture of widely-spaced and high radial ribs, aperture rounded, columellar lamella well developed, peristome thickened and expanded. 


\section{Etymology}

We named this new species "khwantongae" in memory of Ms. Kanita Khwantong, who made the first discovery of this new species.

\section{Distribution}

This species is known only from the limestone hills in Chonburi and Rayong Provinces.

\section{Taxon discussion}

The shell shape of Diplommatina is generally fusiform or tower-shaped with mostly low and strong radial ribs, which are formed as tubular projections in some species and mostly rounded or angular periphery whorls. For example, the shell of $D$. hidagai is fusiform covered with low and strong radial ribs or the shell of $D$. nimannandhi Panha et al., 2002 has tower-shaped, radial ribs forming as semi-tubular peripheral projections. In this new species, Diplommatina khwantongae sp. $\mathrm{n}$. is a very distinct species of epitoniid snail due to its cylindrical, thin radial ribs and shouldered whorls.
(1) D. burapha spec. nov.
2 D. chadathongae spec. nov.
(3) D. chanthaburi spec. nov.
(4) D. crispata khaochamaoensis
5 D. fusiformis spec. nov.
6 D. khwantongae spec. nov.

Figure 5. doi

Distribution of Diplommatina spp. in Eastern Thailand. Code of location shown in Table 1. 


\section{Discussion}

In Thailand, a total of 18 Diplommatina species have been reported (Inkhavilay et al. 2019,Panha and Burch 2005, Tongkerd et al. 2013). From that, there is only one species, Diplommatina crispata khaochamaoensis recorded in eastern Thailand. This work increases the number of species reported in the eastern part of Thailand to five species and makes a total of 22 species of the Diplommatina in Thailand. These data imply that eastern Thailand might be a hot spot for the Diplommatina. Previous research on this microsnails' group reveals the high endemism in almost every species due to their ecology, living on the limestone hill that limits their dispersal ability (Panha and Burch 2005). In this study, the species described also show high endemism except for the Diplommatina crispata khaochamaoensis, which occurred in almost all study sites. (Fig. 5)

\section{Acknowledgements}

This work was supported by a Research Grant to Burapha University through the National Research Council of Thailand (Grant no. 136/2561). We thank all the students in the Malacology Laboratory for their generous help in the field survey. Thanks are also due to the anonymous reviewers for their helpful suggestions for improving this manuscript.

\section{References}

- Inkhavilay K, Sutcharit C, Bantaowong U, Chanabun R, Siriwut W, Srisonchai R, Panha $S$, et al. (2019) Annotated checklist of the terrestrial molluscs from Laos (Mollusca, Gastropoda). ZooKeys 834: 1-166. https://doi.org/10.3897/zookeys.834.28800

- Maassen WJ (2001) Four new Diplommatinidae (Gastropoda, Prosobranchia, Diplommatinidae) from southern Thailand and northern Peninsular Malaysia. Basteria 65 (1/3): 51-56.

- $\quad$ Panha S (1996) Two new species of Diplommatina from Thailand (Prosobranchia: Diplommatinidae). Walkerana 8 (19): 41-47.

- Panha S, Burch JB (1996) New species of Diplommatina from Thailand (Prosobranchia: Diplommatinidae). Walkerana 8 (19): 49-62.

- Panha S, Kanchanasaka B, Burch JB (1998) New taxa of Diplommatina from Thailand (Prosobranchia: Diplommatinidae). Walkerana 9 (22): 153-170.

- $\quad$ Panha S, Burch JB (2001) Two new species of Diplommatina from Thailand (Prosobranchia: Diplommatinidae). Tropical Natural History 1 (1): 33-37.

- Panha S, Burch JB (2005) An introduction to the microsnails of Thailand. Malacological Review 37 (38): 37-155.

- $\quad$ Tongkerd P, Sutcharit C, Panha S (2013) Two new species of micro land snails from two islands in the Andaman Sea (Prosobranchia: Diplommatinidae; Pulmonata: Pupillidae). Tropical Natural History 13 (2): 65-76. 